(3\%) had a NRTI mutation, six (3\%) had at least one nNRTI mutation and $4(2 \%)$ had a PI mutation. There were no dual/triple class/INI mutations. There was no significant change in the prevalence of TDR over the study period. The overall prevalence of non-B subtype was 53/212(25\%;95\% $\mathrm{CI}=19.6-31.2$ ), and was not more frequently seen in non$\mathrm{UK}$ born individuals $(\mathrm{OR}=1.24 ; \mathrm{CI}=0.66-2.33, \mathrm{p}=0.51)$. Patients with TDR were older [45.v.36 years, $\mathrm{p}=0.006$ ] and have non-B subtype $(\mathrm{OR}=2.96 ; \mathrm{CI}=1.08-8.13, \mathrm{p}=0.03)$. Although overall rates of bacterial STIs was high (34\%), having a bacterial STI was not associated with TDR $(\mathrm{OR}=1.77 ; 95 \% \mathrm{CI}=0.66-4.82, \mathrm{p}=0.26)$.

Conclusion TDR is associated with age and non-B subtype in our population. HIV TDR is not decreasing locally and remains a small but significant concern despite effective HIV prevention strategies, which may not reach hidden populations affected by HIV. Continued efforts to reduce HIV transmission must target hidden populations and we must maintain adequate surveillance of TDR.

\section{P026 SEXUALLY TRANSMITTED SHIGELLA FLEXNERI AND SHIGELLA SONNEI IN MEN WHO HAVE SEX WITH MEN}

1,2D Richardson* , 'J Devlin, ${ }^{1} \mathrm{C}$ Fitzpatrick, ${ }^{1} \mathrm{~N}$ Pinto-Sander. 'Brighton and Sussex University Hospitals Nhs Trust, Brighton, UK; ${ }^{2}$ Brighton and Sussex Medical School, Brighton, UK

10.1136/sextrans-2021-sti.173

Shigella outbreaks in MSM are associated with sexual networks involving transmission of other sexually transmitted infections (STIs), geosocial app use for meeting sexual partners and chemsex. There is increasing antimicrobial resistance (AMR) in Shigella amongst MSM. We aimed to describe cases and patterns of AMR amongst MSM with Shigella between 2016-2019.

There were 33 cases of shigellosis in MSM identified, with a median age of 38-years (IQR 34-47). 11/33(33\%) reported recent chemsex use, $14 / 33(42 \%)$ were HIV positive. Of the 19/33 HIV negative MSM, 7/19(37\%) were using HIV preexposure prophylaxis. The mean number of sexual partners in the previous 3 months was six. 15/33(45\%) had Shigella sonnei, 5/33(15\%) Shigella flexneri, and 13/33(39\%) were DNAPCR positive but culture negative. 14/33(42\%) were diagnosed with at least one other STI. MSM with Shigella flexneri were more likely to be HIV positive than Shigella sonnei $(\mathrm{p}<0.05)$. Antimicrobial sensitivities were only available in $11 / 15$ cases of Shigella sonnei[fully sensitive(9\%), resistant to ciprofloxacin (9\%), resistant to azithromycin(36\%), resistant to ciprofloxacin and azithromycin(45\%)]. 7/33(21\%) were treated presumptively on the day of presentation with intramuscular ceftriaxone $2 \mathrm{~g}$ for $1-3$ days followed by oral ciprofloxacin. No MSM with ciprofloxacin resistance received ciprofloxacin; the remaining 26/33 (78\%) did not receive antimicrobials and their diarrhoea resolved.

We have shown that Shigella flexneri is more frequently seen in HIV positive MSM and is associated with chemsex, and resistance to both azithromycin and ciprofloxacin is common in Shigella sonnei. The increasing use of PCR culture independent diagnostic tests make it more difficult to identify cases and clusters within sexual networks in MSM of multidrug resistant shigellosis: clinicians and microbiologists should be vigilant when managing MSM with diarrhoea to prevent large outbreaks of highly resistant shigellosis.

\section{P027 HOW WOULD SEXUAL HEALTH CLINICIANS MANAGE FIRST ATTENDANCE OF MEN WHO HAVE SEX WITH MEN WITH ANO-GENITAL ULCERS?}

1,2,3 D Richardson ${ }^{*}{ }^{1} \mathrm{~N}$ Pinto-Sander, ${ }^{3} \mathrm{P}$ Kumar, ${ }^{3} \mathrm{~T}$ Jaricha, ${ }^{3} \mathrm{~J}$ Walsh, ${ }^{3,4} \mathrm{D}$ Lewis, ${ }^{1}$ Brighto and Sussex University Hospitals NHS Trust, Brighton, UK; ${ }^{2}$ Brighton and Sussex Medical School, Brighton, UK; ${ }^{3}$ Western Sydney Sexual Health Centre, Parramatta (Western Sydney Local Health District), Australia; ${ }^{4}$ University of Sydney Medical School and Marie Bashir Institute of infectious Diseases and biosecurity, Westmead Clinical School, Westmead, Australia

\subsection{6/sextrans-2021-sti.174}

Background There has been a significant increase in infectious syphilis in men who have sex with men(MSM). Primary syphilis classically presents as painless ano-genital ulceration however recent data suggests that up to $50 \%$ of lesions are painful. We aimed to discover current clinical practice when experienced sexual health clinicians are faced with MSM presenting with ano-genital ulcers.

Method An anonymous online survey was sent to clinicians in Brighton, UK and Western Sydney, Australia.

Results Twenty-nine sexual health clinicians (17 doctors and 12 nurses) from the UK (Brighton) and Australia (Sydney) responded to an anonymous online survey; (16/29,55\%) had been working in sexual heath for more than ten years. In the absence of dark-field microscopy, when faced with a scenario of a non-penicillin allergic MSM with a 5-day history of a large painless penile ulcer, 26/29(90\%;95\% CI $=78.6-100.7)$ would treat presumptively on the day. Most $(21 / 29,72 \% ; 95 \%$ $\mathrm{CI}=56.1-88.7)$ would treat MSM presumptively for syphilis with a large painful anal ulcer. Sexual health clinicians are more likely to offer empirical treatment based upon (1) clinical history (number of sexual partners, previous syphilis, sexual contacts of syphilis, PrEP use, HIV positivity, chem-sex), (2) clinical examination (confidence with clinical diagnosis or having 'classical features' of syphilis) or (3) patient factors (vulnerability factors, less likely to return for treatment or abstain from sex), and are less likely to offer presumptive treatment if they have penicillin allergy, a painful lesion and acceptable laboratory reporting times.

Conclusion In the absence same day highly sensitive assays, experienced sexual health clinicians are managing MSM with ano-genital ulcers based upon sexual history, clinical examination and patient factors. The turn-around-times for laboratorybased assays are vital for syphilis control and prevention. To reduce infectious syphilis, we need to improve access to real time, ideally point-of-care, molecular assays for detection of Treponema pallidum in ano-genital ulcer samples.

\section{P028 SYMPTOMATIC LYMPHOGRANULOMA VENEREUM (LGV) PROCTITIS IN MEN WHO HAVE SEX WITH MEN (MSM)}

${ }^{1,2}$ D Richardson*, ' J Devlin, 'Z Buss, ${ }^{1} \mathrm{C}$ Fitzpatrick, ${ }^{1} \mathrm{~N}$ Pinto-Sander. 'Brighton and Sussex University Hospitals Nhs Trust, Brighton, UK; ${ }^{2}$ Brighton and Sussex Medical School, Brighton, UK

\subsection{6/sextrans-2021-sti.175}

Lymphogranuloma venereum (LGV) is a sub-type of Chlamydia trachomatis and is associated with proctitis. LGV proctitis is associated with recreational drug use particularly chemsex, HIV, hepatitis C, and other sexually transmitted infection transmission. The presenting symptoms of LGV proctitis are not well characterised. We aimed to describe the rectal 